\title{
Study on the Outcome of Treatment of Hyperuricemia with Febuxostat in Patients with Chronic Kidney Disease
}

\author{
Article by Colin Alphonse ${ }^{1}$, M. P. Biju Vareed ${ }^{2}$ \\ ${ }^{1}$ Department of Medicine, Jubilee memorial hospital, Trivandrum, Kerala \\ ${ }^{2}$ Department of Nephrology, District Cooperative Hospital, Kozhikode \\ Email: colinalphonse@gmail.com ${ }^{1}$
}

\begin{abstract}
Hyperuricemia, previously thought as a consequence of renal impairement, has been recently identified as an independent risk factor for the development of Chronic Kidney disease. This study was done to find out the effect of treatment of hyperuricemia in CKD patients. This observational study was conducted at Jubilee memorial hospital and Dr sm csi medical college, Trivandrum. The study population included 200 patients with CKD with hyperuricemia from the department of General medicine and department of Nephrology. Detailed history, anthropometry and clinical examination were recorded from all patients and serum uric acid levels and GFR before and 3 months after treatment were compared. Other study parameters were analysed within the CKD group. Treatment response of hyperuricemia with febuxostat among various stages of CKD was observed. Comparing prevalence hyperuricemia among different stages of CKD after 3month treatment with febuxostat, there was significant association between different stages of CKD and uric acid ( $p$ value $=0.000$ ). The present observational study suggests that febuxostat may slow the progression of mild-to-moderate CKD. Prevalence of hyperuricemia is significantly higher in chronic kidney diseases patients, especially stages 3 and 4. Uric acid level is significantly higher in overweight CKD subjects.
\end{abstract}

Keywords: Hyperuricemia, CKD, GFR.

\section{Introduction}

Although high uric acid level is often seen in CKD patients, it was not clear whether hyperuricemia per se is an individual risk factor in development and progression of CKD. Uric acid is the final oxidation product of purine metabolism and is renally excreted ${ }^{1}$.Studies have shown that increasing levels of uric acid causes NAPDH-dependent oxidative changes which promote apoptosis $^{2}$. This finding shows connection between hyperuricemia and tubulointerstitial renal damage. It also causes afferent renal arteriopathy and tubulointerstitial fibrosis through reninangiotensin-aldosterone mechanism ${ }^{3}$.

Febuxostat is a novel xanthine oxidase inhibitor that is safe for CKD patients due to its hepatic elimination. Several studies ${ }^{4,5,6}$ have reported the renoprotective effects of febuxostat, and recently, febuxostat has been reported to improve renal function in patients with CKD stage $3^{7}$.

This study was undertaken to estimate the response of treatment of hyperuricemia with febuxostat in patient with CKD. It also evaluated the various clinical, demographic and social factors associated with hyperuricemia in CKD patients.

\section{Aim}

1. to estimate renoprotective effect of febuxostat.

2. To evaluate the various clinical, demographic and social factors associated with hyperuricemia in CKD patients.

\section{Materials and methods}

Type of study: Hospital based observation study. The study was undertaken in the Department of Medicine and Department of Nephrology, Jubilee memorial hospital, Trivandrum and Dr SM CSI Medical college and Hospital, Trivandrum, kerala. 
DOI: 10.21522/TIJMD.2013.07.01.Art006

ISSN: $2520-3118$

Duration of study: 3 months

Inclusion criteria: Patients aged >18 years and diagnosed as chronic kidney disease sepeiwith hyperuricemia

\section{Exclusion criteria}

1. Stage 1 and stage 5 CKD and HD Patients

2. Patients with gout.

3. Patients on drugs affecting serum uric acid levels (thiazide diuretics, pyrazinamide, allopurinol)

4. Post renal transplant patients.

5. Patient on chemotherapy.

6. Pregnant women.

7. Patients with malignancy

8. Patients with chronic liver disease

\section{Maneuver}

Subjects were selected for the study based on the inclusion, exclusion criteria. Patients with CKD with hyperuricemia were considered as cases

1. A Pre tested semi structure questionnaire used for collecting socio demographic history and clinical details of subjects.

- Height: Height was measured with the help of a metric isepiscale attached to a vertical board.s[epi

- Weight: Dial type bathroom scale weighing machine was used for weight measurement. Weight was measured in kilogram. isep:

- Bodymassindex (BMI): Calculated using Quetelet index.

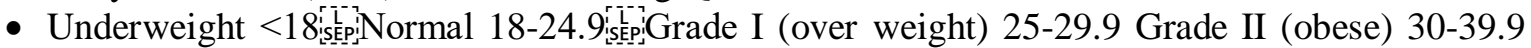
Grade III (very obese) $>40$ istep

- Blood pressure recording: $\mathrm{BP}$ is recorded in the sitting posture in the right upper limb using a proper sized cuff. iscep:

- Coronary artery disease: Cases are said to have coronary artery disease with any one of the following.

- Past history of coronary artery disease with a sicepidocumented evidence istepi

- ECG changes of past or present myocardial infarction is ispiconfirmed by an echocardiography by an experienced iscepicardiologist. istepi]

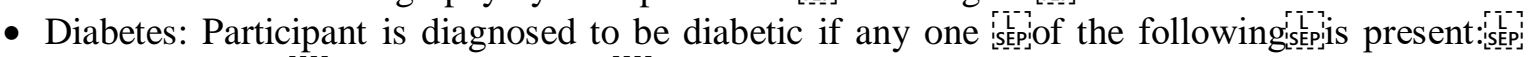
FBS $>126 \mathrm{mg} / \mathrm{dl}$ lisep,, PPBS $>200 \mathrm{mg} / \mathrm{d}$ liseper Patient already on anti-diabetic drugs.

-

- Detailed history regarding patient's education, occupation, family income, daily physical activities, smoking, alcohol intake and family history of hypertension were asked.

2. Lab investigations included(a) serum creatinine, (b) serum uric acid, (c) FBS, (d) PPBS, (e)HBA1C, (f) TROP I, (g) FLP, (h) ECG, (i)ECHO, (j) Blood routine and Chest X ray.

\section{Statistical analysis}

Collected information will be entered in Excel Spread Sheet in Computer and appropriate statistical package will be used for analysis. Data were analyzed using SPSS statistical software and proportion, mean and standard deviations were calculated. Statistical tests like chi square test and $\mathrm{t}$ test will be used for testing differences

\section{Results}

Study group consisted of $200 \mathrm{CKD}$ with hyperuricemia patients. Among the cases 136 were males and 64 were females. Age distribution to be given-30 to 75

The mean uric acid level in among CKD group was $10.6( \pm 1.978 \mathrm{SD}) \mathrm{mg} / \mathrm{dl}$ and it was not statistically significant with $\mathrm{P}<0.439$. Mean uric acid level among the CKD patients in different age groups were compared as shown. The age groups and their mean uric acid values were 30-45, 46-60 and 61-75 and 9.76, 9.99 and10.21 $\mathrm{mg} / \mathrm{dl}$ respectively. There was no significant relationship 
between age and uric acid levels in this study. $(\mathrm{p}=0.586)$ sepep.

Mean uric acid levels in males and females were 10.07 and10.03 $\mathrm{mg} / \mathrm{dl}$ respectively. Though Mean uric acid level is slightly higher in males than females, this was not statistically significant. $(\mathrm{p}=0.908)$.

Mean serum uric acid in smokers and non-smokers in males was 9.76 and $10.23 \mathrm{mg} / \mathrm{dl}$ respectively. Though mean uric acid level in non-smokers was higher than smokers in this study group, it was not statistically significant $(\mathrm{p}=0.182)$

In this study among non-alcoholic and alcoholics, the mean uric levels were 10.03 and 10.18 respectively. Though alcoholic patients had slightly higher mean uric acid level, this was not statistically significant $(\mathrm{p}=0.695)$.

The mean uric acid levels among normal, overweight and obese were 8.80,10.12 and10.06 mg/dl respectively. Mean serum uric level was increased in overweight population though it was not statistically significant $(\mathrm{p}=0.244)$.

Table 1. Influence of uric acid on GFR before treatment

\begin{tabular}{|l|l|l|l|l|l|l|l|l|l|l|l|}
\hline \multicolumn{10}{|l|}{ Basal Uric Acid } & Total \\
\hline GFR & 7 & 8 & 9 & 10 & 11 & 12 & 13 & 14 & 15 & 16 & \\
\hline 2 & 1 & 0 & 0 & 0 & 0 & 0 & 0 & 0 & 0 & 0 & 1 \\
\hline 3 A & 1 & 9 & 15 & 3 & 4 & 5 & 0 & 4 & 0 & 1 & 42 \\
\hline 3 B & 5 & 22 & 32 & 31 & 28 & 4 & 6 & 4 & 1 & 4 & 137 \\
\hline 4 & 1 & 2 & 6 & 3 & 3 & 3 & 0 & 1 & 0 & 1 & 20 \\
\hline Total & 8 & 33 & 53 & 37 & 35 & 12 & 6 & 9 & 1 & 6 & 200 \\
\hline
\end{tabular}

Table 2. Influence of uric acid on GFR after treatment with febuxostat

\begin{tabular}{|l|l|l|l|l|l|l|l|l|l|l|l|}
\hline Uric Acid After 3 Month Treatment with Febuxostat \\
\hline GFR & 4 & 5 & 6 & 7 & 8 & 9 & 10 & 11 & & Total \\
\hline 2 & 1 & 7 & 17 & 4 & 1 & 0 & 1 & 0 & & 31 \\
\hline 3A & 6 & 22 & 35 & 24 & 10 & 6 & 2 & 0 & & & 105 \\
\hline 3B & 2 & 22 & 14 & 17 & 4 & 2 & 1 & 0 & & 62 \\
\hline 4 & 0 & 1 & 0 & 0 & 0 & 0 & 0 & 1 & & & 2 \\
\hline Total & 9 & 52 & 66 & 45 & 15 & 8 & 4 & 1 & & & 200 \\
\hline
\end{tabular}

\section{$P$ VALUE $=0.000$}

Table 3. Parameters influencing serum uric acid level in CKD

\begin{tabular}{|l|l|l|l|}
\hline Parameters & & Serum uric acid & P value \\
\hline \multirow{4}{*}{ Age } & & & \\
& $30-45$ & 9.76 & 0.586 \\
\cline { 2 - 3 } & $46-60$ & 9.99 & \\
\cline { 2 - 3 } & $61-75$ & 10.21 & \multirow{2}{*}{0.908} \\
\hline \multirow{2}{*}{ Sex } & M & 10.07 & \\
\cline { 2 - 3 } & F & 10.03 & 0.181 \\
\hline Smoking & YES & 9.76 & \\
\hline
\end{tabular}


DOI: $10.21522 / \mathrm{TIJMD} \cdot 2013.07 .01 . A r t 006$

ISSN: $2520-3118$

\begin{tabular}{|c|c|c|c|}
\hline & No & 10.19 & \\
\hline \multirow[t]{2}{*}{ Alcohol } & Yes & 10.18 & \multirow[t]{2}{*}{0.695} \\
\hline & $\mathrm{No}$ & 10.03 & \\
\hline \multirow[t]{3}{*}{ Bmi } & $18.5-24.99$ & 8.80 & \multirow[t]{3}{*}{0.241} \\
\hline & $25-29.99$ & 10.12 & \\
\hline & $30-35$ & 10.06 & \\
\hline \multirow[t]{2}{*}{ Hypertension } & Yes & 10.22 & \multirow[t]{2}{*}{0.102} \\
\hline & No & 9.74 & \\
\hline \multirow[t]{2}{*}{ Diabetes } & Yes & 9.97 & \multirow[t]{3}{*}{0.562} \\
\hline & $\mathrm{No}$ & 10.33 & \\
\hline \multirow[t]{3}{*}{ Acs } & No & 9.64 & \\
\hline & & & \multirow[t]{2}{*}{0.472} \\
\hline & Yes & 10.08 & \\
\hline Dyslipidemia & No & 10.06 & 0.940 \\
\hline
\end{tabular}

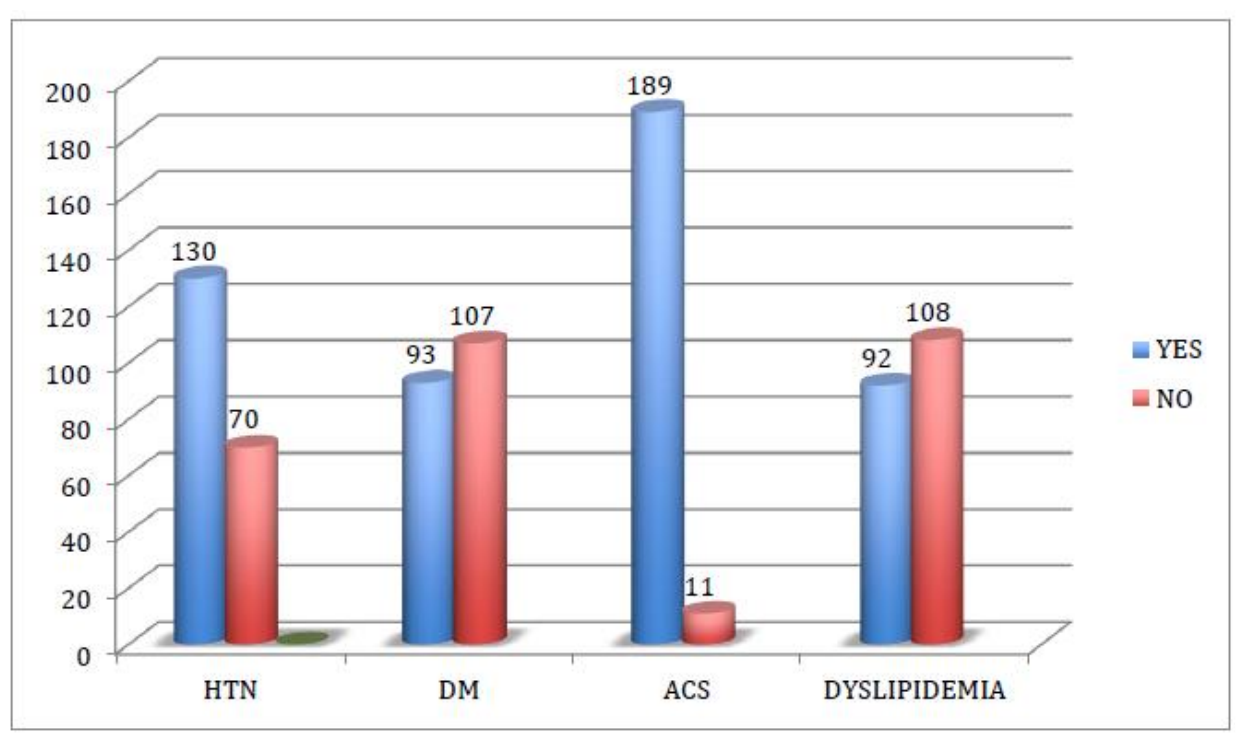

Figure1. Prevalence of various conditions in CKD

Conclusion: The present observational study suggests that febuxostat may slow the progression of mild-to-moderate CKD. Additional large sample-size RCTs are required to determine the long-term renoprotective effects of febuxostat in hyperuricemic patients with CKD. Prevalence of hyperuricemia is significantly higher in chronic kidney diseases patients, especially stages 3 and 4 . Uric acid level is significantly higher in overweight CKD subjects.

\section{References}

[1].Johnson, R. J., Lanaspa, M. A., \& Gaucher, E. A. (2011). Uric acid: a danger signal from the RNA world that may have a role in the epidemic of obesity, metabolic syndrome, and cardiorenal disease: evolutionary considerations. Seminars in Nephrology, 31(5), 394-399.

[2].Mazzali M, Kanellis J, Han L, et al. Hyperuricemia induces a primary renal arteriolopathy in rats by a

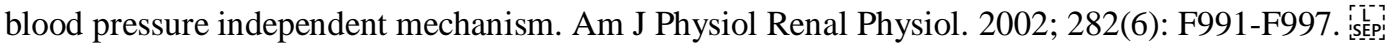

[3].Omori, H., Kawada, N., Inoue, K., Ueda, Y., Yamamoto, R., Matsui, I., ...Rakugi H. (2012). Use of xanthine oxidase inhibitor febuxostat inhibits renal interstitial inflammation and fibrosis in unilateral ureteral obstructive nephropathy. Clin Exp Nephrol, 16,549-556. https://doi.org/10.1007/s10157-012-0609. 
[4].Sezai, A., Soma, M., Nakata, K., Hata, M., Yoshitake, I., Wakui, S., ... Shiono, M. (2013). Comparison of febuxostat and allopurinol for hyperuricemia in cardiac surgery patients (NU-FLASH Trial) Circ J,77,20432049. https://doi.org/10.1253/circj.CJ-13-0082.

[5]. Sircar, D., Chatterjee, S., Waikhom, R., Golay, V., Raychaudhury, A., Chatterjee, S., \&Pandey, R. (2015). Efficacy of febuxostat for slowing the GFR decline in patients with CKD and asymptomatic hyperuricemia: a 6-month, double-blind, randomized, placebo-controlled trial. Am J Kidney Dis,66,945-950. https://doi.org/10.1053/j.ajkd.2015.05.017.

[6].Tanaka, K., Nakayama, M., Kanno, M., Kimura, H., Watanabe, K., Tani, Y., ... Watanabe, T. (2015). Reno-protective effects of febuxostat in hyperuricemic patients with chronic kidney disease: a parallel-group, randomized, controlled trial. Clin Exp Nephrol,19,1044-1053. https://doi.org/10.1007/s10157-015-1095-1.

[7].Verzola, D., Ratto, E., Villaggio, B., Parodi, E. L., Pontremoli, R., Garibotto, G., \& Viazzi, F. (2014). Uric acid promotes apoptosis in human proximal tubule cells by oxidative stress and activation of NAPDH oxidase NOX 4. PloS ONE,9(12). https://doi.org/10.1371/journal.pone.0115210. 